\title{
PER2 Variation is Associated with Diurnal Preference in a Korean Young Population
}

\author{
Heon-Jeong Lee $\cdot$ Leen Kim $\cdot$ Seung-Gul Kang $•$ \\ Ho-Kyoung Yoon $\cdot$ Jung-Eun Choi $\cdot$ \\ Young-Min Park $\cdot$ Seog Ju Kim $\cdot$ Daniel F. Kripke
}

Received: 15 April 2010/Accepted: 9 September 2010/Published online: 8 October 2010

(C) The Author(s) 2010. This article is published with open access at Springerlink.com

\begin{abstract}
The PER2 gene has been reported to influence diurnal preference. In this study, we have attempted to characterize the associations between the PER2 gene polymorphisms and diurnal preference in a population of healthy young subjects, controlling for the social and environmental confounding factors. Subjects were 299 students in a college, carefully selected to be mentally and physically healthy. All subjects completed the 13-item composite scale for morningness (CSM). PER2 gene polymorphisms were genotyped by PCR-based methods. Genotype and allele carrier status of a PER2 G3853A polymorphism (rs934945) were associated with CSM scores. Carriers of the $3853 \mathrm{G}$ allele showed significantly higher CSM scores $(P=0.004$, $P=0.009$, and $P=0.001$; total, morningness, and activity plan, respectively). There were no significant differences on
\end{abstract}

Edited by Tatiana Foroud.

H.-J. Lee · L. Kim $(\bowtie) \cdot$ S.-G. Kang · H.-K. Yoon · J.-E. Choi

Department of Psychiatry, Korea University College

of Medicine, 126-1, 5-Ka, Anam-Dong, Sungbuk-Ku,

Seoul 136-705, Korea

e-mail: kuhjnv@naver.com

Y.-M. Park

Department of Psychiatry, Inje University College of Medicine, Goyang, Korea

\section{S. J. Kim}

Department of Psychiatry, Gachon University of Medicine and Science, Incheon, Korea

D. F. Kripke

Scripps Clinic Sleep Center, La Jolla, CA, USA

H.-J. Lee · J.-E. Choi

Division of Brain Korea 21, Korea University

College of Medicine, Seoul, Korea
CSM scores among genotypes and allele status of PER2 rs2304672. This result indicates that rs934945 of PER2 may be associated with diurnal preference in a Korean healthy population.

Keywords Diurnal preference - Polymorphism - PER2 . Morningness

\section{Introduction}

Diurnal preference is an attribute reflecting whether people are alert and preferring to be active either early or late in the day. It also referred to as chronotype or morningness/eveningness trait. Morning types awaken early and are most alert in the first part of the day, and evening types are most alert in the late evening hours and prefer to go to bed late. This trait can be evaluated in an individual via the completion of a self-reported questionnaire. Diurnal preference is believed to be related to the phase of endogenous circadian rhythms. Recent genetic findings have demonstrated that certain molecular mechanisms control circadian rhythms. They are driven by cell-autonomous transcriptiontranslation feedback loops in the brain and body (Takahashi et al. 2008), that are orchestrated mainly by a biological clock located in the suprachiasmatic nuclei $(\mathrm{SCN})$ of the anterior hypothalamus (Liu et al. 2007). The SCN is entrained to light-dark cycles and its output influences rhythms in behavior by secreting factors that act locally within the hypothalamus and entrains subservient circadian oscillators in peripheral tissues via humoral signals. Peripheral oscillators are not light sensitive and thus have to be entrained by nonphotic cues such as feeding time (Schibler et al. 2003). In mammals, the core circadian rhythm is regulated by a negative feedback loop, wherein 
the transcription factors $C L O C K$ and NPAS2 during the day form complexes with $A R N T L$ and ARNTL2 that bind DNA and activate the transcription of PERI/PER2 and CRYI/ $C R Y 2$ genes. Heterodimerized PER and CRY proteins translocate to the nucleus and block their own transcription by interaction with CLOCK/NPAS2-ARNTL/ARNTL2 heterodimers. The PER-CRY complexes are gradually phosphorylated to be degraded. These dynamic interactions of molecular signals translate into reliably-recurring cellular events, including changes in membrane potentials. Such signals can then be transmitted to connecting neurons, which control physiological processes governed by the circadian rhythms.

In previous genetic studies for human diurnal preference, the $3111 \mathrm{C} / \mathrm{T}$ variation of the $C L O C K$ gene was the first human clock-gene variation reported to affect diurnal preference (Katzenberg et al. 1998). This finding was confirmed by one research group (Mishima et al. 2005), but not by another (Robilliard et al. 2002). Lee et al. (2007) could not replicate this association but found an interaction between the CLOCK and the $\mathrm{G}$ protein $\beta 3$ subunit gene (rs5443) on diurnal preference in healthy young Korean sample.

Carpen et al. (2005) reported that the $111 \mathrm{G}$ allele frequency of PER2 (rs2304672) was significantly higher in subjects with extreme morning preference compared to those with extreme evening preference. The $\mathrm{C} 111 \mathrm{G}$ single nucleotide polymorphism (SNP) was also reported to be associated with advanced sleep phase syndrome (ASPS) in Japanese ASPS families (Satoh et al. 2003). Also, 111G allele was nominally associated with bipolar disorder (Kripke et al. 2009). PER2 SNP 10870 was associated with morningness in winter depression patients (Partonen et al. 2007).

Because diurnal preference is influenced by age, gender and environmental factors, we carefully selected a relatively homogeneous sample population composed of young Korean medical college students. They were almost all of similar age and shared the same regular environmental schedule in the same medical college. Ethnically, Koreans are relatively genetically homogeneous and have less population stratification problem.

We chose two potentially functional PER2 SNPs (rs934945 and rs2304672) for the candidate markers for diurnal preference after considering the previous reports and minor allele frequencies in Korean population.

\section{Methods}

\section{Subjects}

The participants in this experiment included 299 unrelated Korean medical college students $(\mathrm{M}=191, \mathrm{~F}=108)$, with a mean age of $22.9(\mathrm{SD}=2.14$, range of age $=18-32)$ years. All of these volunteers were recruited from the Korea University College of Medicine in Seoul, Korea. Some participants had been included in a previous study (Lee et al. 2007). Individuals with family histories of substance abuse or major psychiatric disorders (e.g., schizophrenia or mood disorders) were excluded. All participants completed unstructured clinical interviews, which were given by welltrained psychiatrists. We excluded students with major medical or psychiatric problems. All students were residents of Seoul. All study protocols were approved by the Ethics Committee of Korea University, and all participants signed their informed consent before being enrolled into the study.

\section{Genotyping}

Genomic DNA was extracted from leukocytes using a QIAamp Blood Kit (Qiagen, Germany) according to the manufacturer's protocol, and genotyping was performed by methods described below.

\section{PER2}

The G3853A SNP (rs934945), a coding region SNP, results in a nonsynonymous amino-acid substitution (Gly/Glu) in the protein. This SNP was detected using PCR amplification and restriction digestion, using PCR primers $5^{\prime}$-CTC AGC GAA GTG TCG GAC ACC-3' and 5'-TCA TCT CTT CAA GCA CCA CC- $3^{\prime}$. The polymorphism is recognized by digestion with BamHI (Fermentas, Canada) (Malison et al. 2006) using following amplification conditions: $95^{\circ} \mathrm{C}$ for $5 \mathrm{~min}$, then 30 cycles of $95^{\circ} \mathrm{C}$ for $30 \mathrm{~s}$, $61.5^{\circ} \mathrm{C}$ for $30 \mathrm{~s}$, and $72^{\circ} \mathrm{C}$ for $30 \mathrm{~s}$. Agarose gel electrophoresis was used to identify whether individuals were heterozygous or homozygous for either of the alleles.

For genotyping C111G SNP (rs2304672), primers were designed using a web-based allele specific primer (http:// bioinfo.biotec.or.th/WASP). The wild-type (WT) forward primer was $5^{\prime}$-TGT TTG CCA GCT TCG TCC-3'. The mutant (MT) forward primer was $5^{\prime}$-TGT TTG CCA GCT TCG TCG- $3^{\prime}$. The universal reverse primer was $5^{\prime}$-GTT GGT CTC ATG TCC ACT- $3^{\prime}$. A mismatch at position 1 of the $3^{\prime}$ end (underlined base) was introduced into the WT and the MT forward primers, respectively.

\section{Diurnal preference assessment}

Diurnal preference was measured by a single administration of the 13-item composite scale for morningness (CSM) (Smith et al. 1989). The CSM is considered to be psychometrically as sound as Horne and Östberg's Morningness-Eveningness Questionnaire and shorter in length (Greenwood 1994). Randler and Diaz-Morales 
(2007) identified a three-factor solution in large-scale college student samples. Three subscales were labeled 'morningness' (items 1, 3, 6, 8, 10, 11), 'activity plan' (items 2, 7, 9, 13), and 'morning alertness' (items 4, 5, 12). Volunteers were asked to complete the Korean version of the CSM, the reliability of which was verified by Yoon et al. (1997). Among 13 items, 3 of the items are graded on a 5-point scale from 1 to 5 , and the remaining 10 items are graded on a 4-point scale, from 1 to 4 . Therefore, the total score ranges from 13 to 55 . Higher scores indicate a morning preference, and lower scores indicate an evening preference.

\section{Statistical analysis}

Analyses of covariance (ANCOVAs) using gender as a covariate were performed in order to determine effects of genotype and allele carrier status on mean CSM scores and scores from its three subscales (morningness, activity plan, and morning alertness). A two-tailed alpha of 0.05 was chosen. A correction for multiple testing was not performed, because of the explorative approach to a genetically complex trait in which a phenotype-genotype relationship has not been established, and such corrections may inappropriately increase type II error rates (Rothman 1990). All statistical tests were carried out using SPSS for Windows.

\section{Results}

The mean \pm SD score on the CSM scale for all subjects was $31.90 \pm 6.39$. There was no significant difference in total CSM score between gender groups, although females showed a trend towards higher morningness score (31.40 \pm 6.02 for males vs. $32.80 \pm 6.93$ for females; $t=1.83, P=0.069)$. The genotype distributions of the two polymorphisms in PER2 were not in deviation from Hardy-Weinberg equilibrium. Minor allele frequencies of PER2 rs2304672 in our sample were relative low, $8.4 \%$. However, that of PER2 rs934945 was $36.5 \%$.

Table 1 showed total CSM score and three factor subscores in subject groups classified by two polymorphisms of PER 2 circadian genes. In addition to genotypic comparisons, we performed comparison according to the allele carrier status referencing previous study results. The $F$ and $P$ values in Table 1 were outcomes of ANCOVAs with gender as a covariate. There were no significant differences among genotypes and allele carrier status for PER2 C111G (rs2304672). However, PER2 G3853A SNP (rs934945) showed significantly different CSM scores and its three subscales among the genotype and allele carrier status. CSM scores were significantly associated according to the G3853A genotypes $(P=0.012, P=0.031$, and $P=0.003$; total, morningness, and activity plan, respectively). The
Table 1 The composite scale for morningness scores in subject groups classified by two PER2 polymorphisms (ANCOVA with sex as a covariate)

\begin{tabular}{lllll}
\hline & CSM score & & \\
\cline { 2 - 5 } & Morningness & Morning alertness & Activity planning & Total \\
\hline PER2 C111G rs2304672 & & & & \\
GG $(n=3)$ & $17.67 \pm 7.57$ & $8.67 \pm 1.53$ & $10.33 \pm 6.03$ & $36.67 \pm 14.98$ \\
CG $(n=44)$ & $16.36 \pm 3.05$ & $7.36 \pm 1.74$ & $8.73 \pm 2.19$ & $32.45 \pm 5.55$ \\
CC $(n=252)$ & $15.51 \pm 3.51$ & $7.35 \pm 1.78$ & $8.89 \pm 2.28$ & $31.75 \pm 6.40$ \\
$F$ & 1.76 & 0.84 & 0.65 & 1.20 \\
$P$ & 0.17 & 0.43 & 0.52 & 0.30 \\
$G$ carrier $(n=47)$ & $16.45 \pm 3.36$ & $7.45 \pm 1.74$ & $8.83 \pm 2.50$ & $32.72 \pm 6.30$ \\
$G$ non-carrier $(n=252)$ & $15.51 \pm 3.51$ & $7.35 \pm 1.78$ & $8.89 \pm 2,28$ & $31.75 \pm 6.40$ \\
$F$ & 3.15 & 0.19 & 0.001 & 1.22 \\
$P$ & 0.077 & 0.66 & 0.98 & 0.27 \\
$P E R 2$ G3853A $($ rs934945) & & & & \\
AA $(n=45)$ & $14.40 \pm 3.31$ & $7.11 \pm 1.77$ & $7.84 \pm 2.45$ & $29.36 \pm 6.35$ \\
AG $(n=128)$ & $15.98 \pm 3.77$ & $7.47 \pm 1.78$ & $9.20 \pm 2.44$ & $32.66 \pm 6.89$ \\
GG $(n=126)$ & $15.77 \pm 3.20$ & $7.36 \pm 1.77$ & $8.92 \pm 2.03$ & $32.05 \pm 5.65$ \\
$F$ & 3.52 & 0.63 & 5.79 & 4.47 \\
$P$ & $\mathbf{0 . 0 3 1}$ & 0.54 & $\mathbf{0 . 0 0 3}$ & $\mathbf{0 . 0 1 2}$ \\
$G$ carrier $(n=254)$ & $14.40 \pm 3.31$ & $7.41 \pm 1.77$ & $9.06 \pm 2.25$ & $32.35 \pm 6.30$ \\
$G$ non-carrier $(n=45)$ & $15.88 \pm 3.50$ & $7.11 \pm 1.77$ & $7.84 \pm 2.45$ & $29.36 \pm 6.35$ \\
$F$ & 6.83 & 1.83 & 10.74 & 8.45 \\
$P$ & $\mathbf{0 . 0 0 9}$ & 0.18 & $\mathbf{0 . 0 0 1}$ & $\mathbf{0 . 0 0 4}$ \\
\hline
\end{tabular}


3853G allele carriers received significantly higher CSM scores $(P=0.004, P=0.009$, and $P=0.001$; total, morningness, and activity plan, respectively). However, the morning alertness score was not significantly different by G3853A genotype and allele carrier status.

\section{Discussion}

Our results show that diurnal preference may be related with PER2 G3853A SNP (rs934945). The 3853G allele carrier subjects showed higher morning preference than participants without $3853 \mathrm{G}$ allele in the homogeneous Korean medical students sharing a uniform occupational time schedule. This is the first report that G3853A SNP of PER2 associates with diurnal preference. This G3853A SNP, a coding region SNP, results in a nonsynonymous amino-acid substitution in the protein (Gly/Glu) at position 1244. Therefore there is a possibility that this amino-acid substitution changes PER2 protein function or expression.

In the previous British study, G3853A SNP was tested but no significant relationship between this SNP and diurnal preference was found (Carpen et al. 2005). However, they found the G allele of C111G SNP of PER2 (rs2304672) associated with extreme morning preference. Another group also reported that C111G SNP was associated with advanced sleep phase syndrome (ASPS) having extremely high morningness in two Japanese ASPS families (Satoh et al. 2003). However, we could not find any association between C111G SNP and diurnal preference in our sample. This discrepancy can be explained by a very low $G$ allele frequency in East Asian population. The sample in the Japanese study which reported positive result was only two small pedigrees. What they reported was that $111 \mathrm{G}$ allele was found in three affected or probably affected members of one small familial ASPS pedigree. Similarly, our sample also showed $111 \mathrm{G}$ homozygous subjects have very high mean CSM score compared to other genotypes, though this was statistically not significant because of low frequency $(n=3)$ of $111 \mathrm{G}$ homozygote. If the sample size were larger enough, positive results would be possible.

There was another report presenting a positive result between $P E R 2$ and diurnal preference in an Asian population. One synonymous SNP (G2114A) of PER2 significantly correlated to diurnal preference in a Japanese young healthy population (Matsuo et al. 2007), although 2114A allele showed very low frequency of $4 \%$. We did not explore this SNP as a candidate marker in our study because of its low minor allele frequency in Asian populations.

Among CSM total score and its three factors, only the 'morning alertness' factor score was not significantly different between PER2 G3853A variants. The morning alertness factor reflects degree of alertness, tiredness and recovery after waking up from night's sleep. This result suggests that morning alertness is not particularly influenced by this genetic factor. It might be that morning alertness is more influenced by the quality of night sleep.

Previously, a mutation of PER2 causing a serine to glycine mutation within the casein kinase 1 epsilon (CK1 1 ) binding region of PER2 was reported to cause familial ASPS (Toh et al. 2001). This mutation abolishes one of the phosphorylation target sites for $\mathrm{CK} 1 \varepsilon$ and in vitro experiment and in vivo animal study showed that the mutation reduces CK1 $\varepsilon$-induced phosphorylation of PER2 protein, which impaired the degradation of PER2 proteins in the cytoplasm and advance of the sleep-wake rhythm as a result (Toh et al. 2001; Xu et al. 2007). The G3853A SNP of PER2 which was associated with diurnal preference in our study also causes amino acid change (glycine to glutamine) in PER2 protein. Therefore, the polymorphism may change PER2 protein function. Although an experiment for functional relevance of G3853A SNP is needed, we hypothesized that the G allele carrier of G3853A SNP brings out low PER2 protein activity, which may be associated with higher morningness trait.

Acknowledgment This study was supported by a grant from the Korean Neuropsychiatric Association Research Foundation.

Open Access This article is distributed under the terms of the Creative Commons Attribution Noncommercial License which permits any noncommercial use, distribution, and reproduction in any medium, provided the original author(s) and source are credited.

\section{References}

Carpen JD, Archer SN, Skene DJ, Smits M, von Schantz M (2005) A single-nucleotide polymorphism in the $5^{\prime}$-untranslated region of the $h P E R 2$ gene is associated with diurnal preference. J Sleep Res 14:293-297

Greenwood KM (1994) Long-term stability and psychometric properties of the composite scale of morningness. Ergonomics 37: 377-383

Katzenberg D, Young T, Finn L, Lin L, King DP, Takahashi JS, Mignot E (1998) A CLOCK polymorphism associated with human diurnal preference. Sleep 21:569-576

Kripke DF, Nievergelt CM, Joo EJ, Shekhtman T, Kelsoe JR (2009) Circadian polymorphisms associated with affective disorders. J Circadian Rhythm 7:2

Lee HJ, Paik JW, Kang SG, Lim SW, Kim L (2007) Allelic variants interaction of $C L O C K$ gene and G-protein $\beta 3$ subunit gene with diurnal preference. Chronobiol Int 24:589-597

Liu AC, Welsh DK, Ko CH, Tran HG, Zhang EE, Priest AA, Buhr ED et al (2007) Intercellular coupling confers robustness against mutations in the SCN circadian clock network. Cell 129:605-616

Malison RT, Kranzler HR, Yang BZ, Gelernter J (2006) Human clock, PER1 and PER2 polymorphisms: lack of association with cocaine dependence susceptibility and cocaine-induced paranoia. Psychiatr Genet 16:245-249 
Matsuo M, Shino Y, Yamada N, Ozeki Y, Okawa M (2007) A novel SNP in hPer2 associates with diurnal preference in a healthy population. Sleep Biol Rhythms 5:141-145

Mishima K, Tozawa T, Satoh K, Saitoh H, Mishima Y (2005) The $3111 \mathrm{~T} / \mathrm{C}$ polymorphism of hClock is associated with evening preference and delayed sleep timing in a Japanese population sample. Am J Med Genet B Neuropsychiatr Genet 133B:101-104

Partonen T, Treutlein J, Alpman A, Frank J, Johansson C, Depner M, Aron L et al (2007) Three circadian clock genes Per2, Arntl, and Npas2 contribute to winter depression. Ann Med 39:229-238

Randler C, Diaz-Morales JF (2007) Morningness in German and Spanish students: a comparative study. Eur J Person 21:419-427

Robilliard DL, Archer SN, Arendt J, Lockley SW, Hack LM, English $\mathrm{J}$, Leger D et al (2002) The 3111 Clock gene polymorphism is not associated with sleep and circadian rhythmicity in phenotypically characterized human subjects. J Sleep Res 11:305-312

Rothman KJ (1990) No adjustments are needed for multiple comparisons. Epidemiology 1:43-46

Satoh K, Mishima K, Inoue Y, Ebisawa T, Shimizu T (2003) Two pedigrees of familial advanced sleep phase syndrome in Japan. Sleep 26:416-417
Schibler U, Ripperger J, Brown SA (2003) Peripheral circadian oscillators in mammals: time and food. J Biol Rhythms 18: 250-260

Smith CS, Reilly C, Midkiff K (1989) Evaluation of three circadian rhythm questionnaires with suggestions for an improved measure of morningness. J Appl Psychol 74:728-738

Takahashi JS, Hong HK, Ko CH, McDearmon EL (2008) The genetics of mammalian circadian order and disorder: implications for physiology and disease. Nat Rev Genet 9:764-775

Toh KL, Jones CR, He Y, Eide EJ, Hinz WA, Virshup DM, Ptácek LJ et al (2001) An hPer2 phosphorylation site mutation in familial advanced sleep phase syndrome. Science 291:1040-1043

Xu Y, Toh KL, Jones CR, Shin JY, Fu YH, Ptácek LJ (2007) Modeling of a human circadian mutation yields insights into clock regulation by PER2. Cell 128:59-70

Yoon JS, Shin SM, Kook SH, Lee HY (1997) A preliminary study on the Korean translation of composite scale (KtCS) to measure morningness eveningness. J Kor Neuropsychiatr Assoc 36: $122-134$ 